\title{
A lightweight coin detection and classification algorithm for low quality images
}

\author{
Zhi Liu, Yanru Sun \\ North China University of Technology, Beijing, 100144, China \\ Izliu@126.com
}

Keywords: Coin Detection and Classification; Low Quality Image; Pattern Recognition

\begin{abstract}
Coin detection and classification system is important in banks. When coin images are captured by low end line-scan camera with poor illumination, their quality will be relatively low. A lightweight algorithm is proposed for this kind of images. Firstly, a modified Canny operator is designed to filter vertical line segments to speed up the ellipse fitting steps. Secondly, a rotation invariant feature is applied to describe the feature of a coin. Initial experiments show that the classification accuracy of the proposed algorithm is about $92.6 \%$ for the data given.
\end{abstract}

\section{Introduction}

With the merits of durable and convenient carrying, coins are important circulating currency and play an important role in commodity transaction market. Therefore, coin detection and classification equipment is very important for banks. While various kinds of techniques have been adopted in modern coin detection and classification system, techniques based on images is a popular one ${ }^{[1]}$.

To improve the efficiency of coin detection and classification system, the conveyor belt carrying coins always runs at a relatively high speed, which means the system must capture coin images at a high frame rate. Matrix CCD/CMOS camera captures two dimensional images directly, but cannot capture images at high frame rate, especially for some low end cameras. On the contrary, line-scan digital camera can capture images with relatively high frame rate even with a low cost camera. Therefore, in the designed system, a low end line-scan digital camera is adopted to balance cost and speed.

Different capture equipment produces images with different characteristics, which means different optimal detection and classification algorithm must be designed. Due to the low end line-scan camera adopted and poor illumination, the quality of the images is always relatively low. Figure 1 shows some sample data.

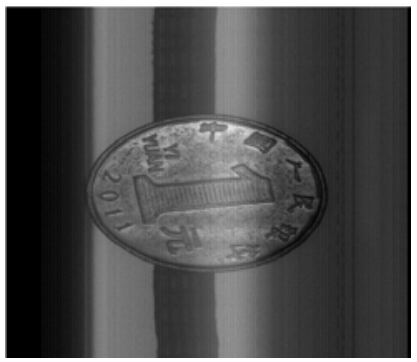

(a) Normal coin

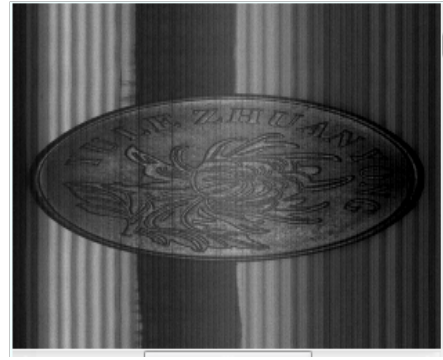

(b) Game coin

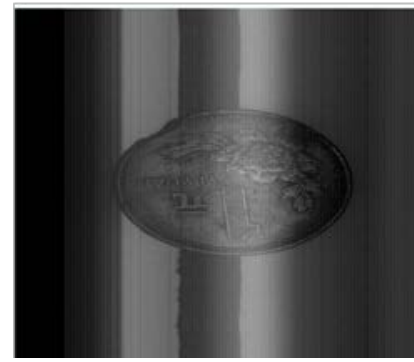

(c) Spoiled coin

Fig.1. Low quality images sampled by the system

In the images showed figure 1, the black strip indicates the conveyor belt. There are three kinds of objects to detect and classify. The first kind is normal coins as is shown in Figure 1(a), which means this kind of coins is genuine and can be put into circulation again. The second one represents counterfeit objects, including coins made for game machine, or some illegally produced fabricate coins. Some counterfeit objects have similar design as genuine coins, one of which is shown in figure 1(b). The third kind is the spoiled coins, which is distributed by the central government bank but is spoiled during the circulating stage. This kind of coins must be recycled by the bank to prevent it to be putted into circulation again. Therefore, the main task of a coin detection and classification system is to distinguish these three kinds of objects and signals the mechanical 
subsystem for further action. From the sample data shown in figure 1, we can find the difficulties of detection and classification coins. (1) Due to the cost budget, the quality of images captured by low end camera is always relatively low. Strips will be always found in the images, which add difficulty to the segmentation of the coin area. (2) The similarity of some game coins to a genuine one is very high. (3) For spoiled coins, there are many kinds of spoiling, such as having holes or grooves in the coin body or worn-out surface. (4) In order to perform real-time processing, the computing complexity of the algorithm must not be too high.

Based on the difficulties mentioned above, we designed a lightweight algorithm for coin detection and classification, which can meet the requirements in classification speed and performance.

\section{Design of the detection and classification algorithm}

The main flow chart of the algorithm is shown in figure 2.

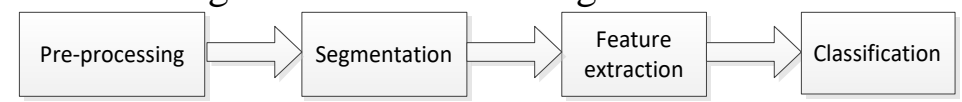

Fig.2. Main flow chart of the algorithm

The pre-processing stage performs some basic processing to input image, such as noise filtering, contrast adjustment, etc..

\section{Area segmentation}

As can be found from figure 1, the area of a coin is mixed with the background. We cannot segment the area of the coins out directly. Since the area of a coin is an ellipse in the captured image, we can find the coin area by detecting an ellipse. The detection procedure is shown in figure 3.

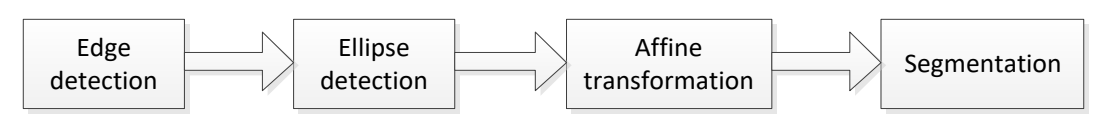

Fig.3. Area segmentation

For edge detection, there are many operators, such as Sobel, Canny, etc.. However, both of them perform not well in our problem. Figure 4(a) shows the result of edge detection using a typical Canny. It can be found that there are too much line segments in the image, which means increase of computation complexity in the following ellipse detection procedure. Since the majority of the edges are vertical line segments, we modified the canny operator by only extract horizontal edges. This process is detailed as the following:

(1) Gaussian smoothing (Suppose $f(x, y)$ is the input image signal, and $H(x, y)$ is the Gaussian filter)

$$
G(x, y)=f(x, y)^{*} H(x, y)
$$

(2) Gradient extraction (Suppose $H_{x}(m, n)$ is a gradient template)

$$
\varphi(m, n)=G(m, n) * H_{x}(m, n)
$$

(3) Non-maximum suppression (The same as that in typical Canny)

(4) Double thresholding (Suppose $m$ is the median value of the image)

$$
\begin{aligned}
t h_{1} & =0.2 \cdot m \\
t h_{2} & =0.4 \cdot m
\end{aligned}
$$

(5) Edge tracking

The result using the modified Canny operator is shown figure 4(b). From the figure, we can find that the majority of the vertical line segments are filtered out by applying the modified canny operator. Therefore, we can use a simpler ellipse fit algorithm to detect the coin area.

After the edge detection process, a dilation and erosion operation is applied, and then a typical ellipse fitting algorithm is applied to detection the coin area[2][3]. The result is shown in figure 5. 


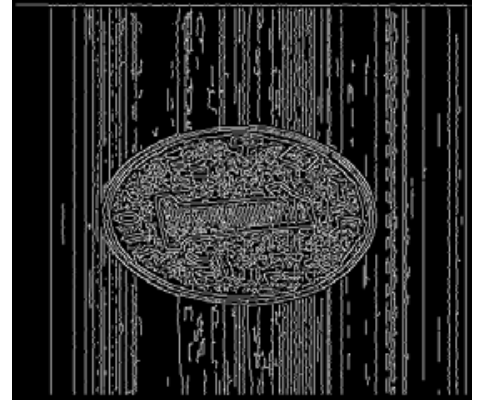

(a) Typical Canny

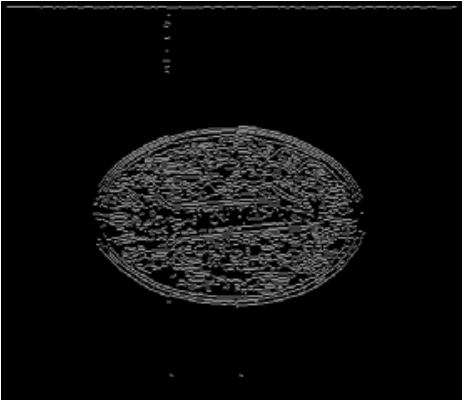

(b) Modified canny

Fig.4. Edge detection

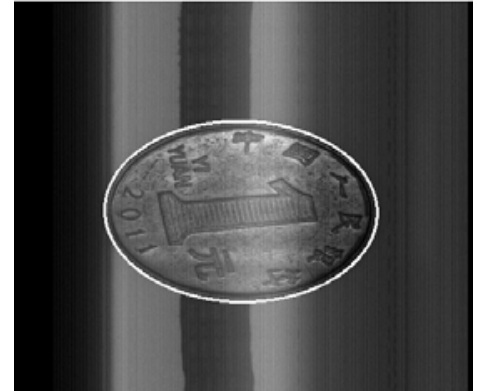

Fig.5. Area detected

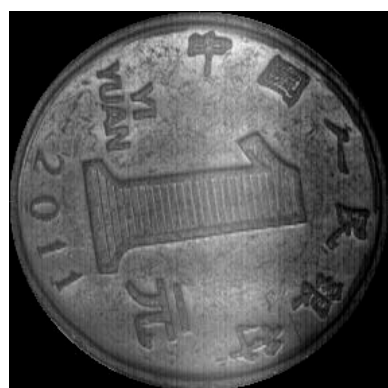

Fig.6. Coin area(affine transformed)

Since the area detected is an ellipse, which is not convenient for feature extraction, it must be transformed into circle. According to the movement of the coin during image capturing, we can do an affine transformation to the ellipse to get a circle. The result is shown in figure 6 .

\section{Feature extraction}

Since the shape of a coin is a circle, the image captured may be at any angle. Therefore, we need to adopt a rotation invariant feature. HOG(Histogram of Oriented Gradient) ${ }^{[4]}$ feature is a popular feature widely used in image pattern recognition. However, HOG feature do not has the property of rotation invariant. To overcome this, many modified HOG feature is proposed ${ }^{[5][6]}$. In this project, we adopt the Rotation invariant Histograms of Oriented Gradients proposed by [6]. The main idea is shown as follows:

(1) Partition the coin area in to concentric annuluses.

(2) Calculate the gradient $\mathbf{g}$ for each point in one annulus.

(3) For each gradient $\mathbf{g}$, calculate the RGT(Radial Gradient Transform) gradient $\left(\mathbf{g}^{T} r, \mathbf{g}^{T} t\right)^{[7]}$

(4) Using equation (5) , (6)to calculate the magnitude and phase of each RGT gradient:

$$
\begin{aligned}
& M=\sqrt{\left(\mathbf{g}^{T} r\right)^{2}+\left(\mathbf{g}^{T} t\right)^{2}} \\
& \theta=\arctan \frac{\mathbf{g}^{T} t}{\mathbf{g}^{T} r}
\end{aligned}
$$

(5) For each annulus, find the histogram of RGT gradient.

(6)Formulate the feature vector by concatenating the histogram from each annulus.

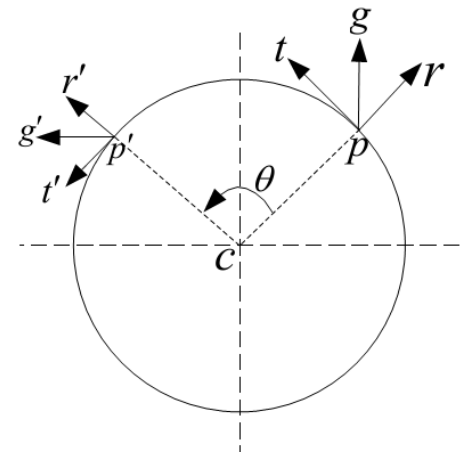

Fig.7. Explaination of RGT gradient 


\section{Classification}

The classification step is performed by using a typical SVM(Supported Vector Machine) classifier. Details of SVM are ignored here due to space limitation.

\section{Experiment results}

Some initial experiments are performed based on the OpenCV library ${ }^{[8]}$. All data is divided into two set, training set and test set. The training set has 800 images, including 300 normal coin images, 300 counterfeit coin images, and 200 spoiled coin images. The test set contains 2000 images, and the test result is listed in Table 1.

Tab.1. Test result

\begin{tabular}{|c|c|c|c|}
\hline Kinds & Normal coins & counterfeit coins & spoiled coins \\
\hline Accuracy & $96.1 \%$ & $92.5 \%$ & $89.3 \%$ \\
\hline
\end{tabular}

The classification accuracy of the proposed algorithm is about $92.6 \%$, at a speed of $25 \mathrm{~ms}$ per image, which can meet the requirements of the system. It can be found from Table 1 that, the correct percentage of spoiled coins is relatively low, this is due to the reason that the feature of spoiled coins may vary greatly, and the classifier tend to classify it to be counterfeit coin if the feature of the testing object do not match normal coins and spoiled coins. Some of the mis-classified images are shown in Figure 8.

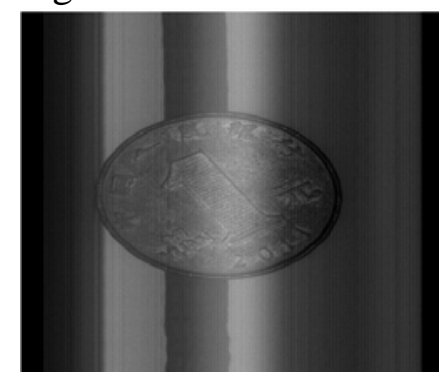

(a)Normal coin classified to be spoiled one

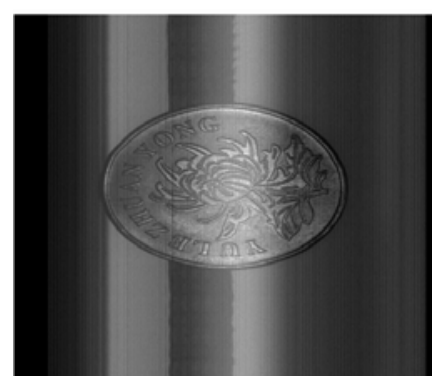

(b) Counterfeit coin classified to be normal one

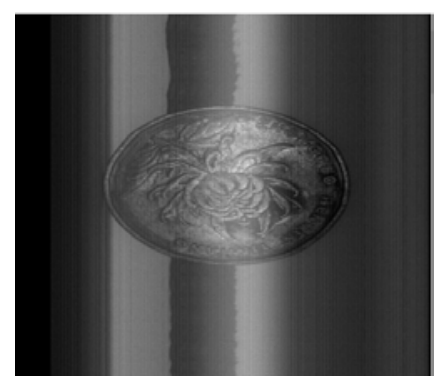

(c) Spoiled coin classified to be counterfeit one

Fig.8. Some mis-classified images

\section{Conclusion}

In this paper, a lightweight coin detection and classification algorithm is proposed. In the proposed algorithm, a modified Canny operator is designed to filter out vertical edge line segments, and an ellipse fit is applied to detection coin area. A rotation invariant feature is adopted to describe different kind of objects. Initial experiments show that the classification accuracy of the proposed algorithm is about $92.6 \%$.

\section{Acknowledgement}

This work is partially supported by the Beijing Municipal Education Commission General Program (KM201610009003)

\section{References}

[1]Vassilas, N. \& Skourlas, C. (2006). Content-based coin retrieval using invariant features and self-organizing maps, Proc. of Int. Conf. on Artif. Neur. Netw., pp. 113-122.

[2] Kalviainen $\mathrm{H}$, Hirvonen P. An extension to the random sized Hough transform exploiting connectivity[J], Pattern Recognition Letters, 1997, 18: 77—85.

[3] Poyato A C, CUEVAS F J M. Polygonal approximation of digital planar curves through break 
point suppression[J]. Pattern Recognition, 2010, 43(1): 14-25.

[4] Navneet Dalal and Bill Triggs. Histograms of Oriented Gradients for Human Detection[A]. Computer Vision and Pattern Recognition[C]. San Diego:Springer, 2005. 886-893.

[5] X. Wang, L. Lin, L. Huang, and S. Yan, "Incorporating Structural Alternatives and Sharing into Hierarchy for Multiclass Object Recognition and Detection”,Proc. IEEE Conf. Comput. Vis. Pattern Recognit. (CVPR), pp.3334-3341, Jun. 2013.

[6] Zhaojie Luo, Jinhui Chen, Tetsuya Takiguchi, Yasuo Ariki. Rotation-invariant histograms of oriented gradients for local patch robust representation. 2015 Asia-Pacific Signal and Information Processing Association Annual Summit and Conference (APSIPA), 2015. 196-199.

[7] Gabriel Takacs. Unified Real-Time Tracking and Recognition with Rotation-Invariant Fast Features[A]. Computer Vision and Pattern Recognition[C]. San Francisco:Springer, 2010. 934-941

[8] opencv.org/downloads.html [EB/OL]. 